\title{
Cobalt Alumino Silicate Ceramic(CASC) Nanocomposite, a Material with Moderately High Dielectric Constant and Low Tangent Loss at a Critical Concentration in High Frequency Range
}

\author{
Biplab Kumar Paul ${ }^{1}$, Smarajit Manna ${ }^{2}$, Debasis Roy ${ }^{1}$, Papiya Nandy ${ }^{1,2}$ and Sukhen Das ${ }^{1,5, *}$ \\ 1 Department of Physics, Jadavpur University. Kolkata-700 032, India \\ 2 Jagadis Bose National Science Talent Search, Kolkata-700 107, India \\ 3 Central Glass and Ceramic Research Institute, Kolkata-700 032, India \\ 4 West Bengal State University, Kolkata, India \\ 5 Indian Institute of Engineering Science and Technology, Shibpur, India \\ * Author to whom correspondence should be addressed; E-Mail: sukhendasju@gmail.com.
}

\section{Published: 4 December 2015}

\begin{abstract}
Cobalt Alumino Silicate Ceramic (CASC) composites, with different molar weight concentration (i.e. $\mathrm{G}_{0}=0, \mathrm{G}_{1}=0.4, \mathrm{G}_{2}=0.6, \mathrm{G}_{3}=0.8, \mathrm{G}_{4}=1.0$, and $\mathrm{G}_{5}=1.2$ (M.W.)) of cobaltus acetate are prepared via sol-gel route. XRD shows mullite and cobalt aluminate phase which is found to depend on the concentrations of $\mathrm{Co}^{+2}$ ions. Field emission scanning electron microscope (FESEM) images show for all samples of CASC nanocomposites after sintering at $1400^{\circ} \mathrm{C}$, nano sized cobalt aluminate grains are embedded in evenly spread mullite grains; but in case of $\mathrm{G}_{0}$, there are only evenly spread mullite grains. The study of dielectric property of the composite samples at room temperature shows that at all concentration $\left(G_{1}, G_{2}, G_{3}, G_{4}\right.$ and $\left.G_{5}\right)$ the dielectric constant is higher than pure mullite $\left(\mathrm{G}_{0}\right)$ and there is a critical concentration of cobaltus acetate (G3) where there is maximum enhancement of dielectric constant in the higher frequency range from $40 \mathrm{KHz}$ to $2 \mathrm{MHz}$. The dielectric constant varies from 44.77 to 37.75 for $\mathrm{G}_{3}$ and from 29.8 to 24.12 for $\mathrm{G}_{0}$ respectively. The tangent loss of composite with $\mathrm{G}_{3}$ has the lowest value than that of other concentrations including pure mullite in the frequency range $40 \mathrm{KHz}$ to $2 \mathrm{MHz}$. Due to high dielectric constant and low tangent loss, the composite with specific concentration and in the high frequency range has great importance as an electronic material.
\end{abstract}

Keywords: Mullite; dielectric constant; tangent loss; electronic material; sensor; capacitor 


\section{Introduction}

During past decades Alumino silicate ceramic composite was used as an advanced structural ceramics and now it is well known as a promising electronic material with some great properties such as high melting point, antierosion, better chemical stability to oxidation as well as good resistance to most chemical attack, great coercivity, good electrical resistance, good mechanical strength, low thermal expansion coefficient, very good high temperature strength and also a low cost material ${ }^{1-4}$.

So this composite have been increasingly gaining importance for high frequency circuit packaging and electronic substrate application due to their low dielectric constant ${ }^{1,5-7}$ since last few decades. But recently researchers have been trying to enhance their dielectric constant by making composites by doping metal ions so that it can maintain its physical properties as earlier and also enhance the electrical and electronic properties. These composites with metal ions have wide range of application in electrical and electronic industries ${ }^{8,9}$. So CASC composite is a leading candidate for high transmitting IR windows, electronic material, humidity sensors, protective coatings with pigments, electrical insulators, and turbine engine components etc ${ }^{10-}$ 13 .

Cobalt aluminate composite behaves like an outstanding dielectric material due to so cobalt aluminate mullite ceramic composites have been studied widely because of its potential applications such as in high charge storage multilayer ceramics capacitors (MLCC), transducer because of its high polarization, high permittivity and very low tangent loss and can be promising candidate in Microelectronic industries, interconnect technology $y^{8,14-17}$..

\section{Experimental}

Chemicals used in the preparation of precursor gels, $\mathrm{C}_{9} \mathrm{H}_{21} \mathrm{O}_{3} \mathrm{Al}_{11}$, puriss (Spectrochem Pvt.Ltd., India.), and $\mathrm{Si}\left(\mathrm{OC}_{2} \mathrm{H}_{5}\right)_{4}$,(MERCK, Germany 99.9\%), , were simultaneously added to $0.5 \mathrm{M}$ solution of $\mathrm{Al}\left(\mathrm{NO}_{3}\right)_{3}, 9 \mathrm{H}_{2} \mathrm{O}$, extra pure(MERCK, India,99.9\%), in double distilled water. The molar ratio of $\mathrm{C}_{9} \mathrm{H}_{21} \mathrm{O}_{3} \mathrm{Al}$ and $\mathrm{Al}$ $\left(\mathrm{NO}_{3}\right)_{3}, 9 \mathrm{H}_{2} \mathrm{O}$ is $7: 2$. In the resulting sol 0.4 $\left(\mathrm{G}_{1}\right), 0.6\left(\mathrm{G}_{2}\right), 0.8\left(\mathrm{G}_{3}\right), 1.0\left(\mathrm{G}_{4}\right)$ and $1.2\left(\mathrm{G}_{5}\right)$ molar weighted $\left(\mathrm{CH}_{3} \mathrm{COO}\right){ }_{2} \mathrm{Co}, 4 \mathrm{H}_{2} \mathrm{O}$, extra pure (MERCK, India 99.9.\%), salt were added to the mixture separately. The amount of given salts for the increasing doping concentration of cobaltus acetate in alumino silicate ceramic system is shown in Table 1.

After stirring the solution for 3 hours, gel formation was completed and was kept overnight at $60^{\circ} \mathrm{C}$ for ageing the solution. The gel was then dried at $120^{\circ} \mathrm{C}$ and after grinding. the samples were then pelletized in cylindrical pellet form of $1 \mathrm{~mm}$ thickness by pressing powder to $70 \mathrm{MPa}$ in a $10 \mathrm{~mm}$ diameter stainless steel die using hydraulic press and sintered in air environment at $1400^{\circ} \mathrm{C}$ for 2 hours at the heating rate of $5^{\circ} \mathrm{C} /$ minute $^{18-20}$. Sintering was performed in air environment only to maintain the neutrality of the sample. Silver paste was painted on both surfaces of the pellet and then dried using hot air flow for 10 to 15 minutes. 
Table 1. Different amount of salts given to prepare CASC composites, with different molar weight concentration.

\begin{tabular}{|c|c|c|c|c|c|c|}
\hline Salts & $\mathbf{G}_{0}$ & $\mathbf{G}_{1}$ & $\mathbf{G}_{2}$ & $\mathbf{G}_{3}$ & $\mathbf{G}_{4}$ & G5 \\
\hline $\mathrm{Al}\left(\mathrm{NO}_{3}\right)_{3}, 9 \mathrm{H}_{2} \mathrm{O}$ & 3.75 & 3.75 & 3.75 & 3.75 & 3.75 & 3.75 \\
\hline $\mathrm{C}_{9} \mathrm{H}_{21} \mathrm{O}_{3} \mathrm{Al}_{11}(\mathrm{~g})$ & 7.30 & 7.30 & 7.30 & 7.30 & 7.30 & 7.30 \\
\hline $\mathrm{Si}\left(\mathrm{OC}_{2} \mathrm{H}_{5}\right)_{4}(\mathrm{~g})$ & 3.40 & 3.40 & 3.40 & 3.40 & 3.40 & 3.40 \\
\hline$\left(\mathrm{CH}_{3} \mathrm{COO}\right)_{2} \mathrm{Co}, 4 \mathrm{H}_{2} \mathrm{O}(\mathrm{g})$ & 0.00 & 1.922 & 2.988 & 3.984 & 4.981 & 5.977 \\
\hline
\end{tabular}

Table 2. Elementary distribution (norm.wt.\%) for all CASC composites.

\begin{tabular}{lllllll}
\hline $\begin{array}{l}\text { Elementary } \\
\text { Distribution. } \\
\text { (In norm.[wt.\%]) }\end{array}$ & $\mathbf{G}_{\mathbf{0}}$ & $\mathbf{G}_{\mathbf{1}}$ & $\mathbf{G}_{\mathbf{2}}$ & $\mathbf{G}_{\mathbf{3}}$ & $\mathbf{G}_{\mathbf{4}}$ & $\mathbf{G}_{\mathbf{5}}$ \\
\hline Aluminum & 42.56 & 43.34 & 42.81 & 30.87 & 32.57 & 19.12 \\
& & & & & & \\
Silicon & 9.22 & 3.96 & 2.58 & 7.82 & 3.51 & 5.66 \\
Cobalt & 0.00 & 9.64 & 13.60 & 24.93 & 30.95 & 51.75 \\
Oxygen & 48.22 & 43.06 & 41.01 & 36.37 & 32.97 & 23.46 \\
\hline
\end{tabular}

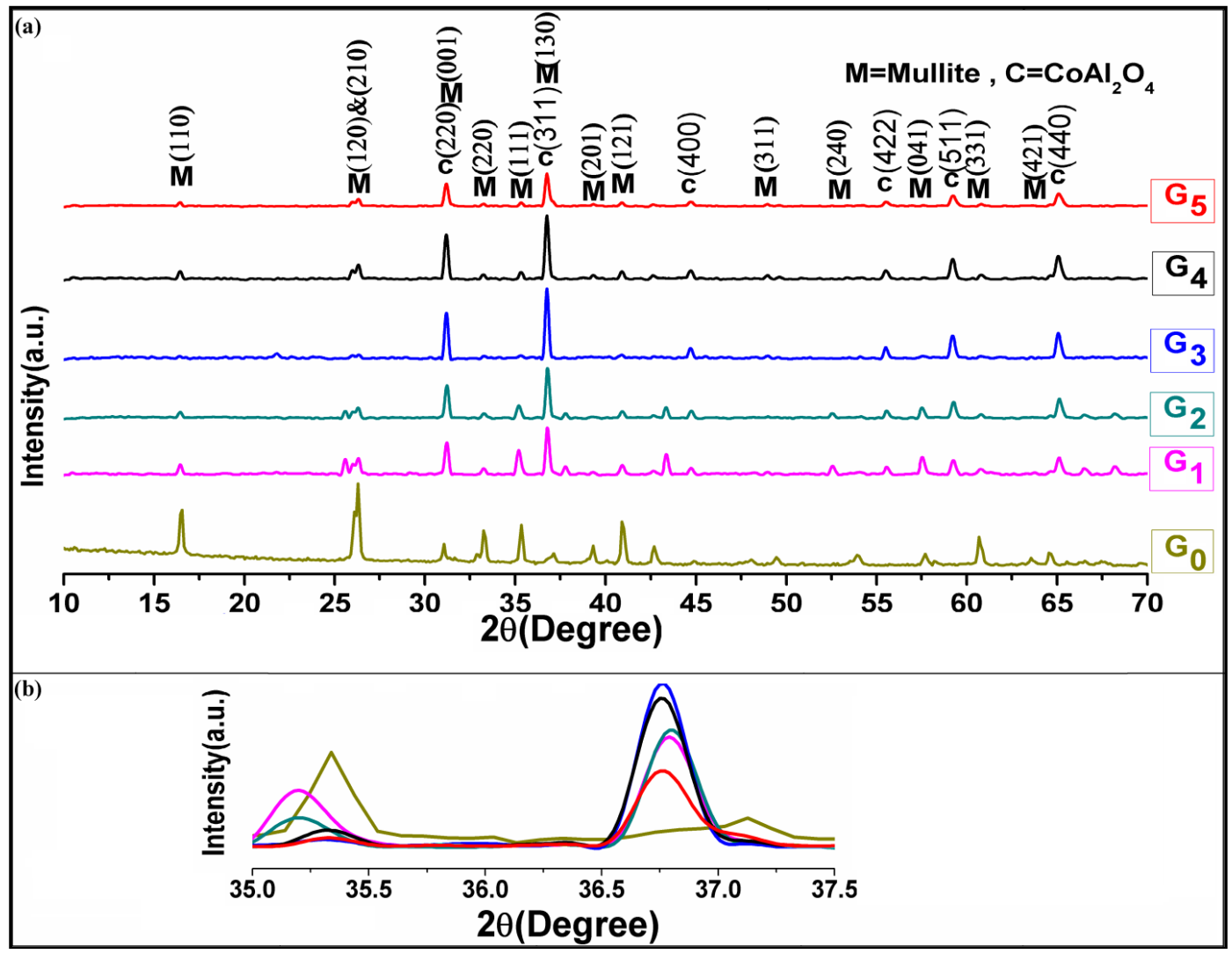

Fig.1 (a).X-ray diffraction pattern of pure mullite and all CASC composites containing increasing doping concentration of Cobaltus Acetate. (b).XRD patterns of composites with different concentration between $2 \theta$ from $35^{\circ}$ to $37.5^{\circ}$. 


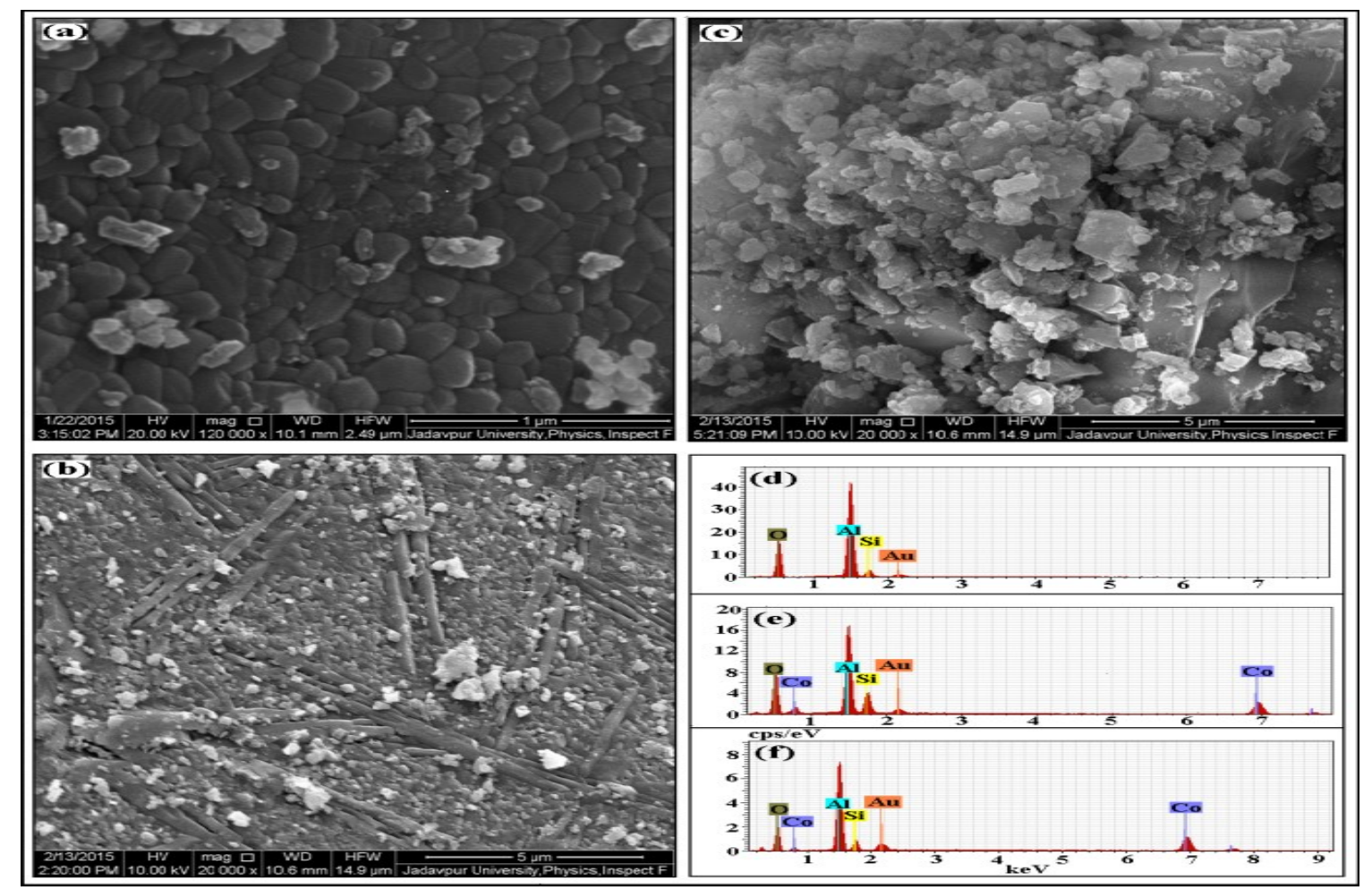

Fig. 2(a),(b) and (c). FESEM micrograph for $\mathrm{G}_{0}, \mathrm{G}_{3}$ and $\mathrm{G}_{5}$ CASC composites respectively. Fig.2(d),(e)and(f). Energy-dispersive X-ray spectroscopy (EDX) image of $\mathrm{G}_{0}$, $\mathrm{G}_{3}$ and $\mathrm{G}_{5} \mathrm{CASC}$ composites respectively.

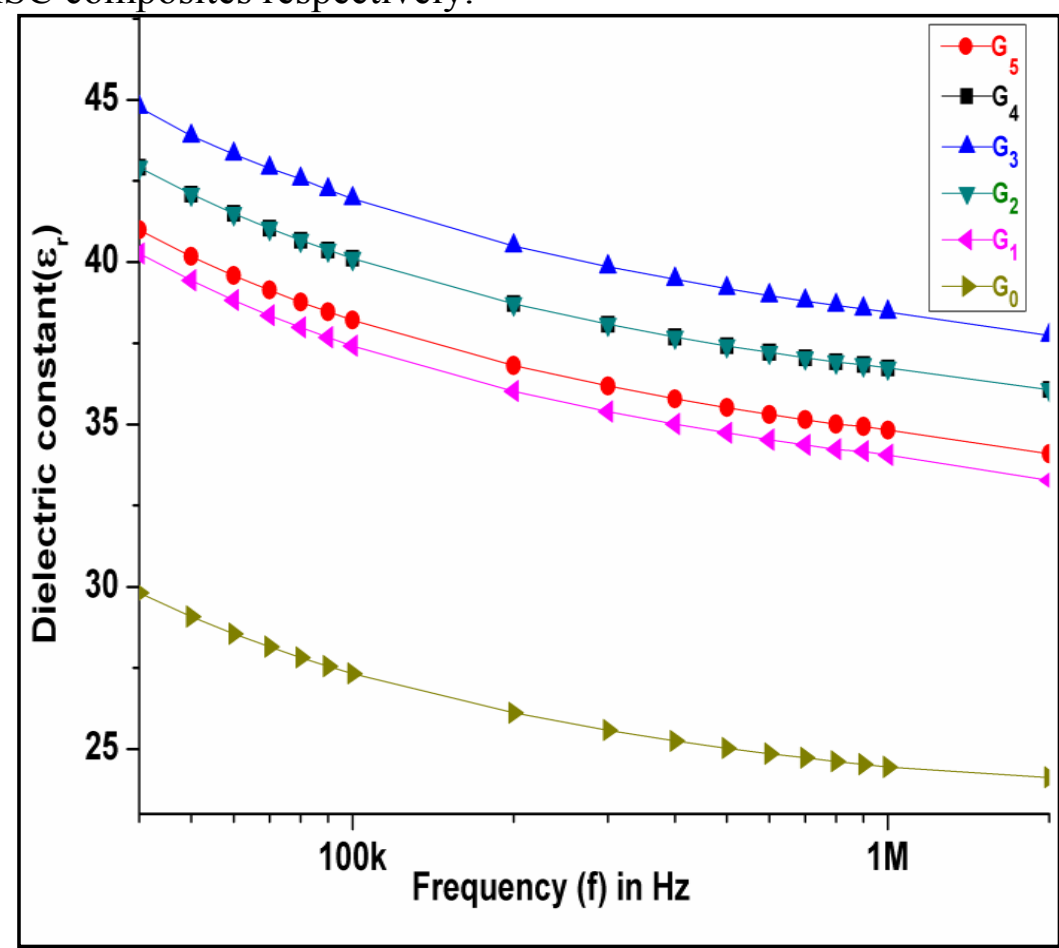

Fig.3. Frequency response Dielectric Constant $\left(\varepsilon_{\mathrm{r}}\right)$ behavior for all CASC composites. 


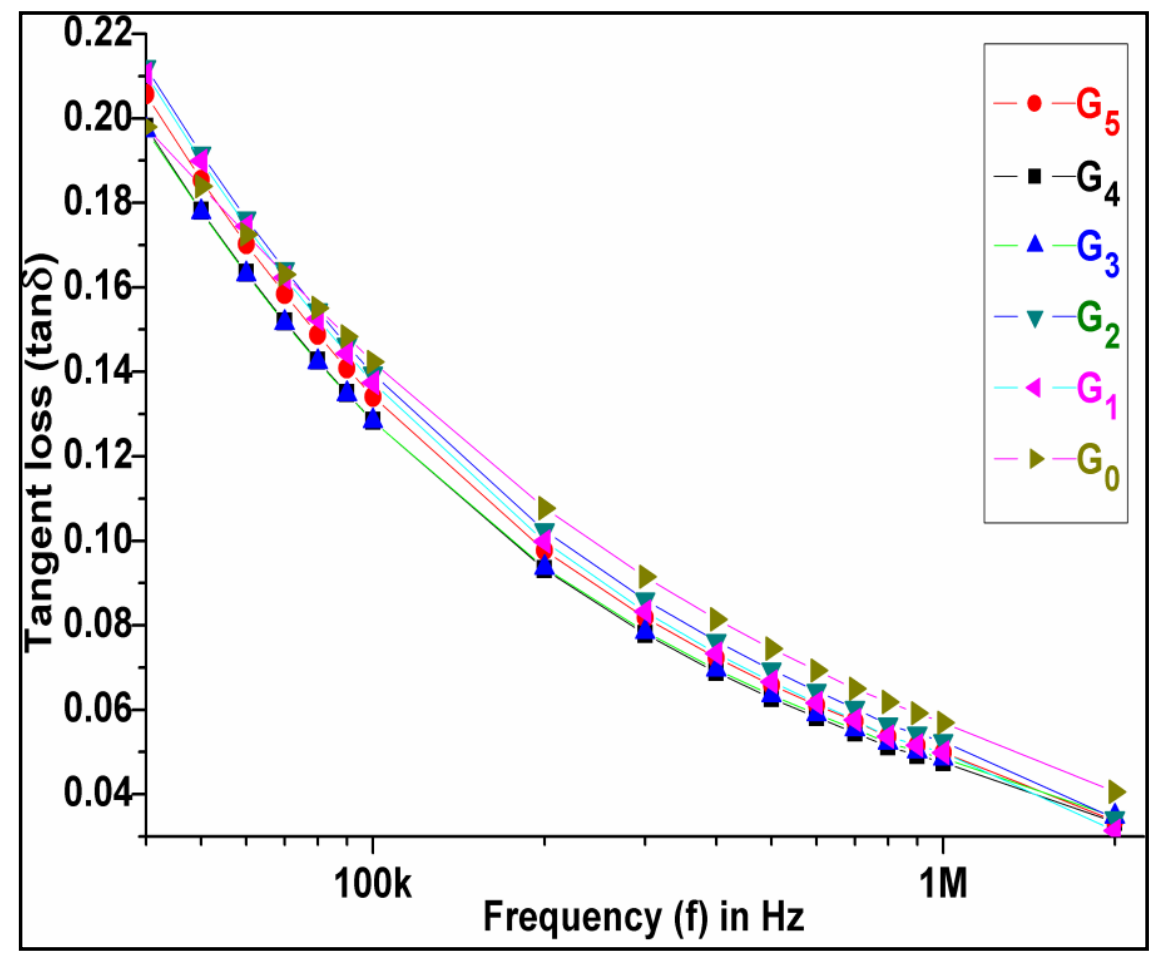

Fig.4. Frequency response Tangent loss (Tan $\delta$ ) behavior for all CASC composites.

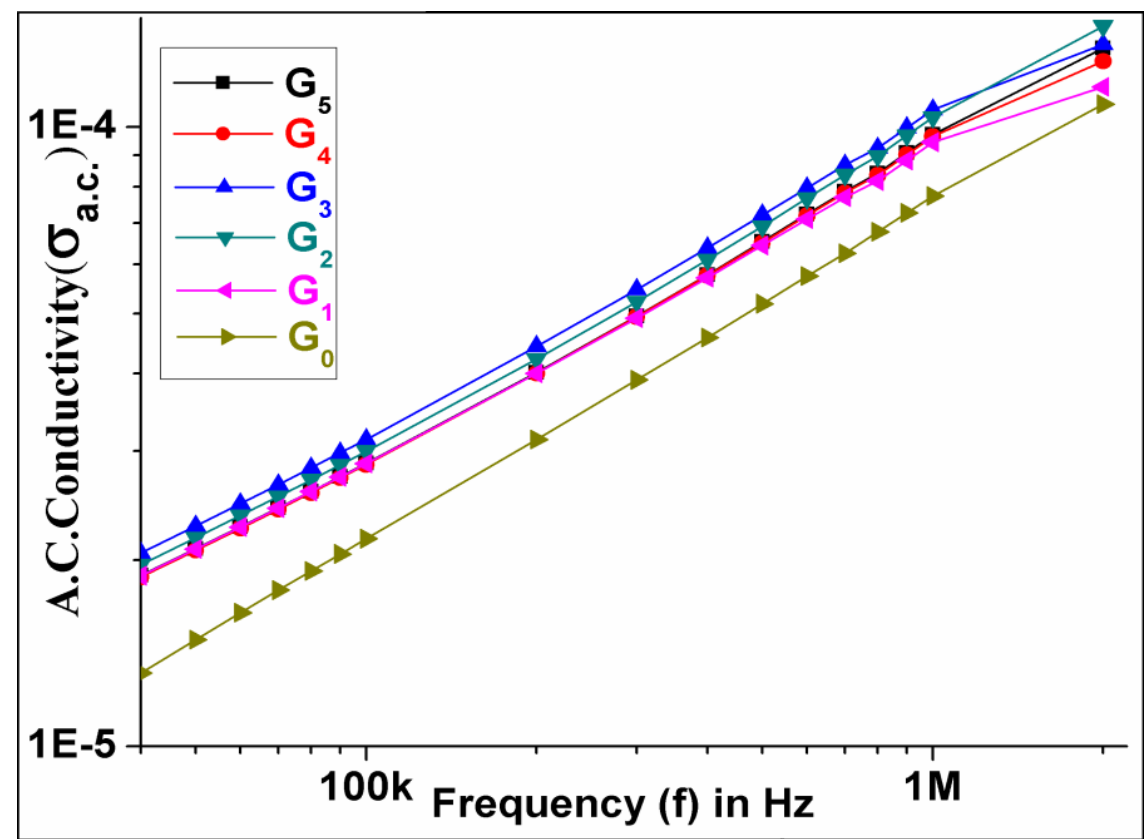

Fig.4. Frequency response of A.C. Conductivity $\left(\sigma_{\text {a.c }}\right)$ for all CASC composites. 


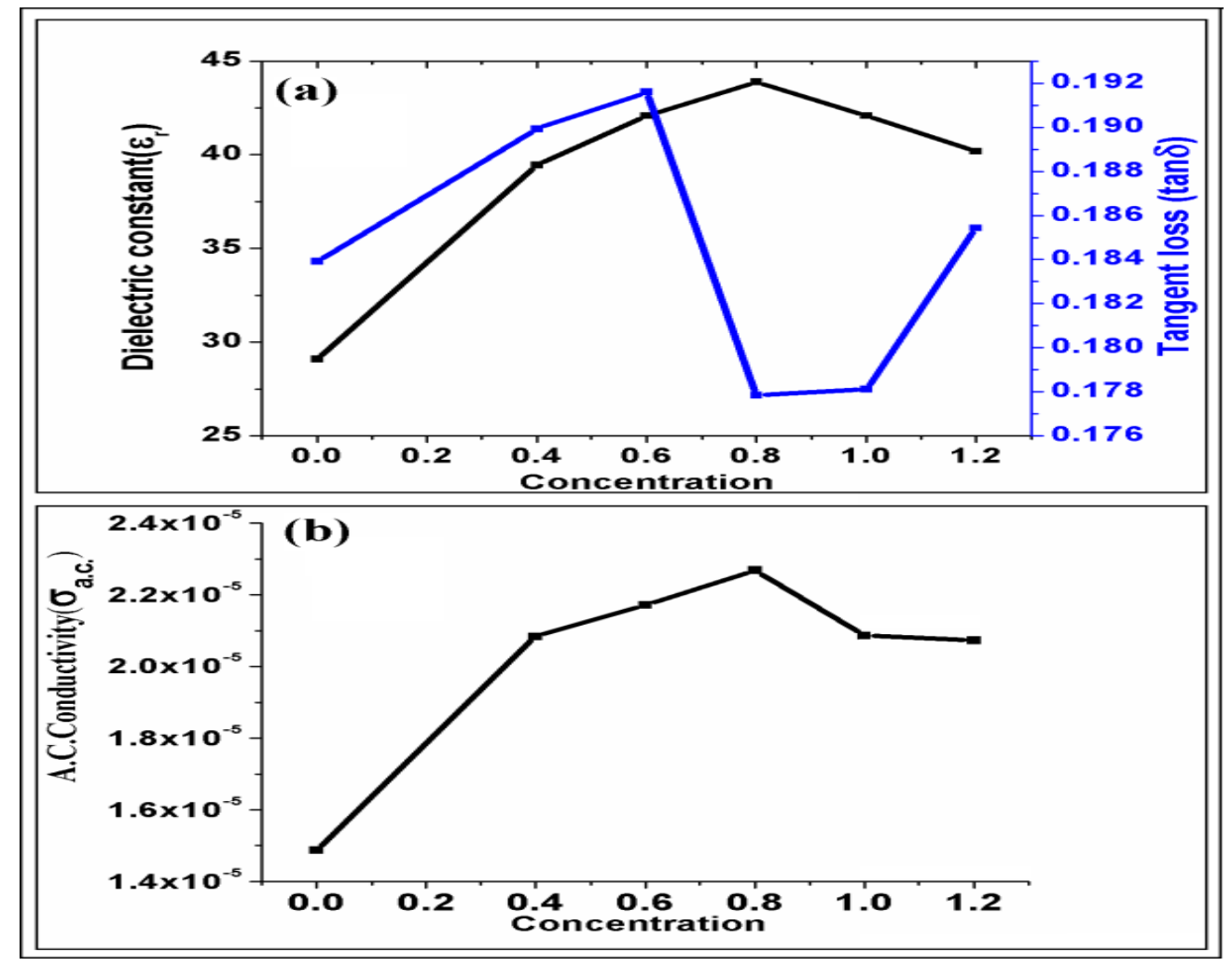

Fig.6.(a) Concentration dependent Dielectric Constant ( $\varepsilon_{\mathrm{r}}$ ) and corresponding Tangent loss (Tan $\delta$ ) and Fig.6.(b) A.C. Conductivity $\left(\sigma_{\text {a.c }}\right)$ behavior for all CASC composites at 50KHz.

The micrographs of mullite composites $\mathrm{G}_{0}$ and $\mathrm{G}_{3}$ and $\mathrm{G}_{5}$ are shown in Figure 2(a) and 2(b) and 2(c) respectively. Micrograph of $\mathrm{G}_{0}$ shows sphere shaped morphology of mullite grains with $100-300 \mathrm{~nm}$ size which is evenly distributed in the whole matrix. On the otherhand micrograph of $\mathrm{G}_{3}$ shows crystalline plate like grains of cobalt aluminate of average size 3-6 $\mu \mathrm{m}$ embedded in uniformly spread numerous smaller mullite particles along with amorphous aggregates 9,18,19,24. For further increased concentration of cobalt salt (i.e. for G5) large amount of agglomerated particles along with bulk cobalt aluminate grains has been observed.

\section{Used Instruments}

The electrical properties of the pellets as a function of frequency were studied at room temperature by using LCR meter (Agilent 4294A precision impedance analyzer $40 \mathrm{~Hz}-110 \mathrm{MHz}$ ) in the frequency range of $40 \mathrm{KHz}$ to $2 \mathrm{MHz}$. X-ray diffractometer (XRD) (Bruker D8 Advanced) was used to identify the crystalline structure and phases of the samples with different concentrations after sintering. The observations were made at angle between $10^{\circ}-70^{\circ}$. The morphology and energy-dispersive X-ray spectroscopy (EDX) of the fracture surface of mortared dust was observed by using field emission scanning electron microscope (FESEM) (FEI Inspect F50).

\section{Results and Discussion}

\subsection{XRD Analysis}

Figure 1(a) and 1(b) shows the XRD spectrum of all CASC composite. Sample $\mathrm{G}_{0}$ shows the 
prominent peaks of a single phase mullite that can be indexed with the standard JCPDS file number (JCPDS Card No-150776). The peaks of $\mathrm{CoAl}_{2} \mathrm{O}_{4}$ emerge when it is added to the mullite. Intensity of the $\mathrm{CoAl}_{2} \mathrm{O}_{4}$ peaks increase with increasing of its concentration in the composites upto $\mathrm{G}_{3}$ and then decrease for higher concentration [peak C (440)]. Intensity of the individual mullite peaks decrease with increasing concentration of cobalt aluminate in the composites [peak M (111)]..

Intensity of the peaks of $\mathrm{CoAl}_{2} \mathrm{O}_{4}$ increases with increasing concentration of cobalt aluminate in the composites upto $\mathrm{G}_{3}$ and then decrease for higher concentration [peak C (311), and C (220)]. Figure 1(b) illustrates the XRD patterns of composites with different concentration between $2 \theta$ of $35^{\circ}-37.5^{\circ}$ showing the strong mullite peaks $M$ (111) and common peaks M (130) and C (311). Decrement of mullite phase with the increasing concentration of cobalt acetate is due to increment of cobalt aluminate phases formed by $\mathrm{Co}^{+2}$ ions. In earlier papers ${ }^{18,19,21,22,24}$, it has been reported that mullite phase increases with the increment of metal ions upto certain concentration and then decreases, same characteristics is reported here. It has been observed here that due to the formation of cobalt the catalytic action of the metal decreases

\subsection{Frequency Dependent Analysis}

\subsubsection{Dielectric Constant Behavior}

The variations of dielectric constant of all CASC composites with frequency are shown in Figure 3. From the figure it is clearly seen that throughout the frequency ranges $40 \mathrm{KHz}$ to 2 $\mathrm{MHz}$, dielectric constant continuously decreases with increasing frequency for all concentration of CASC composites which can be explained by the electron hopping model of Heikes and because of the developed strain within the composite as a result mullite phase decreases with increasing concentration of $\mathrm{Co}^{+2}$ ions. The phase variation in mullite was due to the change of concentration of metal ion and was due to John-Teller distortion. The observed difference in mullite formation of metal ions was due to weak ligand field. For weak ligand field the metal ions will be in high spin configuration. The unpaired electron in the $t_{2 g}$ orbital of $\mathrm{Co}^{2+}\left(\mathrm{d}^{7}\right)$ will cause John- Teller distortion of $\mathrm{CoO}_{6}$ octahedra in the gel matrix.

\subsection{FESEM and EDX Analysis}

Figure 2(d), 2(e) and 2(f) stands for elemental distribution (in normality weight \%) analysis for the sample $\mathrm{G}_{0}$ and $\mathrm{G}_{3}$ and $\mathrm{G}_{5}$ CASC composites respectively. Figure (2)d confirms that there is no cobalt content. Fig 2(e) and 2(f) confirms increasing concentration of cobalt content. As the given cobalt content increases peak intensity of cobalt content also increases. Here $\mathrm{Au}$ content arises from the gold coating unit.

Table 2 shows elementary distribution (norm.wt.\%) for all CASC composites which confirms that with the increasing concentration of cobalt acetate normality weight $\%$ of cobalt also increases.

\section{Electrical Properties Analysis}

Johnston $^{23}$. The effect of polarization is to reduce the field inside the medium. Therefore, the dielectric constant of a substance decreases substantially due to the limited dipole response. As the frequency is increases dipole response is limited and the dielectric constant attained a saturation tendency ${ }^{24}$. In this case the internal individual dipoles contribute the dielectric constant. At comparatively lower frequency range the dipoles can orient easily with electric field and so they can contribute improved 
polarization which is mainly responsible for the enhancement of the dielectric constant ${ }^{23-26 .}$

\subsubsection{Tangent Loss Behavior}

Figure 4 shows the variation of tangent loss with frequency for $\mathrm{G}_{0}$ to $\mathrm{G}_{5}$ in the high frequency range $40 \mathrm{KHz}$ to $2 \mathrm{MHz}$. From the figure it is clearly seen that throughout the whole frequency ranges, tangent loss continuously decreases with increasing frequency for all concentration of CASC composites. At comparatively lower frequency range the dipoles can orient easily with external electric field due to more relaxation time. This phenomenon is mainly responsible for intermolecular friction or vibration which contributes the higher tangent loss. As frequency increases, less polarization effect continues due to less relaxation time. So intermolecular friction or vibration diminishes which is responsible for decreasing tangent loss ${ }^{23-26}$.

5.1.3. Frequency Dependent A.C. Conductivity $\left(\sigma_{\text {a.c }}\right)$ Behavior

A.C. conductivity $\left(\sigma_{\text {a.c }}\right)$ is calculated using the formula,

$\sigma_{\text {a.c. }}=2 \pi \mathrm{f} \tan \delta \epsilon_{\mathrm{r}} \epsilon_{\mathrm{o}} \quad$ Where,

$\sigma_{\mathrm{a} . \mathrm{c}}=\mathrm{AC}$ conductivity,

$\mathrm{f}=$ frequency in $\mathrm{Hz}$,

$\tan \delta=$ tangent loss factor,

$\epsilon_{\mathrm{r}}=$ dielectric constant of the material and

$\epsilon_{\mathrm{o}}=$ vacuum permittivity respectively.

The variation of A.C conductivity with frequency is shown in figure 5. It shows A.C. conductivity increases with frequency. Increase of frequency increased a.c. conductivity by increasing hopping of conducting electrons present in CASC composite. At higher frequency range, rapid increase of conductivity with increasing frequency is referred to electronic polarization effect $^{23-26}$.

\subsection{Concentration Dependent Dielectric}

Constant $\left(\varepsilon_{r}\right)$ and Corresponding Tangent loss

$(\operatorname{Tan} \delta)$ and A.C. Conductivity $\left(\sigma_{\text {a.c }}\right)$ Behabior.

From fig.6(a) it is clearly seen that throughout the frequency ranges $40 \mathrm{KHz}$ to $2 \mathrm{MHz}$, dielectric constant has substantially higher value in case of $G_{1}, G_{2}, G_{3}, G_{4}$ and $G_{5}$ than $G_{0}$ and it also increases almost sharply with increasing concentration of cobalt acetate upto (0.8M.W.\%) concentration i.e. $\left(\mathrm{G}_{3}\right)$, above which it decreases. This phenomenon can be explained by MaxwellWagner-Sillars (MWS) interfacial polarization effect which appears in heterogeneous medium consisting of different phases with different permittivity and conductivity due to accumulation of the charges at the interfaces. At low cobalt concentration (i.e. $\leq 0.8 \mathrm{M} . \mathrm{W} . \%$ ), the well crystalline nano mullite particles are well separated from each other with no such effective interaction between them. On the otherhand well crystalline $\mathrm{CoAl}_{2} \mathrm{O}_{4}$ grains is embedded in good and homogeneously distributed nano crystalline mullite matrix. So the number of nanoparticles and their interfacial area per unit volume increases while the interparticle distance decreases. This improves the average polarization associated with the particles and the coupling between neighboring grains, resulting in the significant enhancement of dielectric constant as well as significant decrement of tangent loss. This phenomenon observed upto $\mathrm{G}_{3}$ i.e.(0.8M.W.\%).

For further increment of cobalt content (i.e.>0.8M.W.\%), the grain size of $\mathrm{CoAl}_{2} \mathrm{O}_{4}$ transformed into bulk form which is dissolved into agglomerated alumino silicate matrix. So the interfacial area per unit volume decreases while the interparticle distance decreases. This 
decreases the average polarization associated with the particles resulting in the further decrement of dielectric constant and ac conductivity as well as increment of tangent loss. This phenomenon is also clearly observed from their microstructures (FESEM) [Fig. 5(a),(b) and (c)].

It is also seen that the value of A.C conductivity is higher for all CASC composite than $\mathrm{G}_{0}$. The increase of $\sigma_{\mathrm{a} . \mathrm{c}}$ is due to the increase of $\mathrm{Co}^{+2}$ ions and their mobility. The electrical conductivity fully depends on the mobility of the metal ions, so for the increasing number of metal ions electrical conductivity is also increased substantially ${ }^{6,24,26}$. Presence of cobalt ions in the mullite matrix increases the mobility of ions, so a.c. conductivity increases with the increasing concentration of cobalt. It has been also observed upto $\mathrm{G}_{3}$ (i.e. $\leq 0.8 \mathrm{M} . \mathrm{W} . \%$ ) concentration ac conductivity increases and for further increasing (i.e.>0.8M.W.\%) concentration ac conductivity decreases. This may be due to more agglomerated glassy phase and amorphous matrix ${ }^{(27-30)}$.

\section{Conclusions}

CASC nanocomposites with different concentrations of cobalt acetate have been synthesized by sol-gel technique and their phase evolution and dielectric properties have been investigated. The dielectric constant of all CASC nanocomposites is higher than alumino-silicate composite throughout the frequency range 40 $\mathrm{KHz}$ to $2 \mathrm{MHz}$ and there is a critical concentration i.e. $\mathrm{G}_{3}(0.8 \mathrm{M}$.W.\%) of metal ions $\left(\mathrm{Co}^{+2}\right)$ where the dielectric constant is maximum. The tangent loss of $\mathrm{G}_{3}(0.8 \mathrm{M} . \mathrm{W} . \%)$ composites is lower than pure alumino-silicate composite in the frequency range $50 \mathrm{KHz}$ and sample $\mathrm{G}_{3}$ has minimum tangent loss in that frequency range. The A.C conductivity increases with frequency for all samples and is higher for metal doped mullite composite than pure alumino-silicate composite for presence of mobile metal ions in the composites. Thus pure alumino-silicate composite which has comparatively low dielectric constant can be modified into materials with high dielectric constant and low tangent loss by making a composite with cobalt acetate with molar weight concentration $0.8 \mathrm{M}$.W.\%. These metal doped mullite composites may be used as dielectric material for the fabrication of high charge storing capacitors and also as ceramic capacitors and can be promising candidate for electronic industries.

\section{Acknowledgments}

We are grateful to UGC, Government of India, for financial assistance. We are also thankful to Dr. Abhijit Kar, Scientific Officer JBNSTS, Kolkata for fruitful discussion.

\section{References and Notes}

1. K. Maex, M.R. Baklanov, D. Shamiryan, F. Lacopi, S.H. Brongersma, Z.S. Yanovitskaya, Appl. Phys., 2003, 93, 8793.

2. H. Schneider, J. Schreuer, B. Hildmann, Journal of the European Ceramic Society, 2008, 28,329.

3. M. I. Osendi \& C. Baudin, Journal of the European Ceramic Society, 1996, 16,211.

4. M.A. Camerucci, G. Urretavizcaya, M.S. Castro, A.L. Cavalieri, J. Eu. Ceram. Soc., 2001, $21,2917$.

5. T. Homma, Materials Science and Engineering, 1998, R23, 243. 
6. T. Kurmara, M. Horiuchi, Y. Takeuchi, S.I. Wakabayashi, Electronic Components and Technology Conference, 1990,1, 68.

7. V. Viswabaskarana, F.D. Gnanama, M. Balasubramanian, App. Clay Sci., 2004, 25, 29.

8. M. M. S. Sanad ,M. M. Rashad ,E. A. Abdel-Aal ,M. F. El-Shahat ,K. Powers, J Mater Sci: Mater. Electron., 2014,25,2487.

9. B. K. Paul, K. Halder, D. Roy, B. Bagchi, A. Bhattacharya, S. Das, J Mater Sci: Mater Electron .DOI 10.1007/s10854-014-2291-6

10. M. Llusar, A.Fores, J.A. Badenes, J. Calbo, M.A. Tena, G. Monros, J. Eur. Ceram. Soc., 2007, $21,1121$.

11. G. Carta, M. Casarin, N. El Habra, M. Natali, G. Rossetto, C. Sada, E .Tondello, P. Zanella, Electrochim. Acta., 2005, 50, 4592.

12. R. R. Turnma ,J.Am. Ceram. Soc., 1991,74,5, 895.

13. I.A. Aksay, D.M. Dabbs And M.Sarikaya, J. Am. Ceram. Soc., 1991,74, 2343.

14. F.Wen, Z. Xu, W. Xia, X. Wei and Z. Zhang, J. Adv.Dielect., 2013, 3, 1350010.

15. B.Bagchi, S.Das, A.Bhattacharya, R.Basu, and P.Nandy, J. Am. Ceram. Soc., 2009, 92,748.

16. S.P.Radhika, K. J. Sreeram, B.U. Nair,J. Adv.Ceram., 2012,1,301.

17. X. Hao, J.Adv.Dielect., 2013, 3,1330001.

18. B.Bagchi, S. Das, A. Bhattacharya, R. Basu, P. Nandy, J Sol-Gel Sci. Technol., 2010, 55,135.

19. D. Roy, B.Bagchi, A.Bhattacharya, S.Das, P.Nandy, J.Wu.Univ.Technol.-Mater. Sci. Ed ., 2012,27,836.

20. K.C.Song, Materials Letters, 1998, 35,290.

21. J.P. Tkalcec, B. G.Eta, S. Kurajica, J. Schmauch, American Mineralogist, 2007,92, 408.

22. W.Lv, Q.Qiu, F.Wang, S.Wei, B.Liu, Z.Luo, Ultrasonics Sonochemistry, 2010, 17,793.

23. R.R. Heikes and W.D. Johnston, Journal of Chemical Physics, 1957, 26, 582.

24. D.Roy, B.Bagchi, S.Das, P. Nandy, Materials Chemistry and Physics, 2013, 138, 375.

25. M.S. Sanad, M.M. Rashad, E.A. Abdel-Aal, M.F. El-Shahat, J. Eur. Ceram. Soc., 2012,32 ,4249.

26. D.Roy, B.Bagchi, S.Das, P.Nandy, J. Electroceram., 2012, 28,261.

27. V. K. Thakur, E. J. Tan, M.F. Linb and P. S. Lee, Polym. Chem.,2011,2,2000.

28. P. Thakur, A. Kool, B. Bagchi, S. Das and P. Nandy, PCCP, DOI: 10.1039/c4cp04006f.

29. C. C. Wang, J. F. Song, H. M. Bao, Q. D. Shen and C. Z. Yang, Adv. Funct. Mater., 2008, 18(8), 1299

30. P. Lunkenheimer, V. Bobnar, A. V. Pronin, A. I. Ritus, A. A. Volkov and A. Loidl, Phys. Rev. B: Condens. Mater. Phys., 2002, 66, 052105.

(C) 2015 by the authors; licensee MDPI, Basel, Switzerland. This article is an open access article distributed under the terms and conditions defined by MDPI AG, the publisher of the Sciforum.net platform. Sciforum papers authors the copyright to their scholarly works. Hence, by submitting a paper to this conference, you retain the copyright, but you grant MDPI AG the non-exclusive and unrevocable license right to publish this paper online on the Sciforum.net platform. This means you can easily submit your paper to any scientific journal at a later stage and transfer the copyright to its publisher (if required by that publisher). (http://sciforum.net/about ). 